\title{
Towards storage rings as quantum computers
}

\author{
K. A. Brown $\odot^{*}$ and T. Roser॰ \\ Brookhaven National Laboratory, Upton, New York 11973-5000, USA
}

(Received 28 February 2020; accepted 4 May 2020; published 13 May 2020)

\begin{abstract}
We explore the possible use of particle beam storage rings as quantum computers. More precisely, we consider creating an ion trap system, in which the same computational basis states can be defined as in a modern ion trap system, but in which the ions have a constant velocity and are rotating in a circular trap. The basic structures that we explore are classical and ultracold crystalline beams. What we propose is a novel method that uses the ion trap quantum computer concept, but puts the ions into a rotating frame of reference. The benefits of this approach are discussed.
\end{abstract}

DOI: 10.1103/PhysRevAccelBeams.23.054701

\section{INTRODUCTION}

A particle accelerator storage ring is an apparatus that stores charged particle beams. The beams, if not cooled, can have very high temperatures and can be treated as classical thermodynamic ensembles of particles confined to some volume. When stored, either as bunches of particles or debunched into a uniform longitudinal (temporal) distribution, the ensemble is in steady state and has constant entropy. In general, such a beam has no specific structure and should act like an ideal gas. However, the particles are necessarily charged and can interact with each other through intrabeam collisions and other phenomena. These processes can cause beam heating, increasing the entropy. In addition, these particle distributions do contain information encoded into the behavior of the beams as they traverse the electromagnetic optics that keep them confined within the storage ring [1-4].

It has been shown that entropy and beam emittance are closely related [5,6], so we will use the term emittance from here on.

To bring a classical particle beam into a regime where quantum mechanical phenomena can be observed, it must be cooled to an extremely low temperature [7]. Conventional beam temperature for a particle beam is defined from the rms single component momentum deviation of the particle distribution. The four dimensional $x, p_{x}, y, p_{y}$ distribution, with $p_{x}$ equal to $\beta \gamma m_{0} c x^{\prime}$, describes a region of space in which the particles are confined as $(u=\{x, y\})$,

\footnotetext{
*kbrown@bnl.gov

Published by the American Physical Society under the terms of the Creative Commons Attribution 4.0 International license. Further distribution of this work must maintain attribution to the author(s) and the published article's title, journal citation, and DOI.
}

$$
\epsilon_{u}=4 \pi\left(\left\langle u^{2}\right\rangle\left\langle u^{\prime 2}\right\rangle-\left\langle u u^{\prime}\right\rangle^{2}\right)^{\frac{1}{2}},
$$

where $\epsilon_{u}$ is the horizonal or vertical beam emittance. We will call the transverse beam temperature the temperature associated with the transverse emittance. Longitudinally, the temperature, $T$, is a function of the momentum spread [8],

$$
\frac{1}{2} k_{B} T=\frac{1}{2} m(\delta v)^{2}
$$

where $\delta v$ is the spread in velocity of the ions in the beam. $k_{B}$ is Boltzmann's constant. In more practical units, temperatures for ion beams can be expressed as,

$$
\begin{gathered}
T_{\|}[K]=\frac{2}{k_{B}}\left(\frac{\delta p}{p_{0}}\right) E_{0}[\mathrm{eV}] \\
T_{\perp u}[K]=\frac{2}{k_{B}}\left(\frac{\epsilon_{u}[m-\mathrm{rad}]}{\left\langle\beta_{u}\right\rangle[m]}\right) E_{0}[\mathrm{eV}],
\end{gathered}
$$

where $T_{\|}$is longitudinal temperature, $T_{\perp u}$ are transverse temperatures, $E_{0}$ is the average ion kinetic energy, and $\left\langle\beta_{u}\right\rangle$ are the average lattice beta functions. Typically, beam dimensions are defined in terms of the rms value from a Gaussian distribution.

There are two basic states of matter that can be created by cooling the beams down to very low temperatures. The first is a classical crystalline beam [9], which is defined, per Wei and Sessler, to be "a cluster of circulating, charged particles in its classical lower-energy state subject to circumferentially varying guiding and focusing electromagnetic forces and Coulomb interacting forces" [7]. The second state of matter is an ultracold crystalline beam, or what we will refer to as an ion Coulomb crystal, that has been cooled to the point where the de Broglie wavelength is greater than or equal to the particle thermal oscillation amplitude, but not 
to the point of the Lamb-Dicke limit [10,11]. This is something of a "Goldilocks" regime, where couplings between internal and external quantum states are not strongly suppressed and is well above a Bose-Einstein condensate [10]. In this regime, thermal vibrations are small enough to distinguish the external quantum modes of the crystalline structure. The term, ion Coulomb crystal, is used by some authors in the ion trap literature [12-14] and is consistent with terms used by other authors $[9,15]$. We purposely choose to use this term since we do not want to imply that we are looking to operate in the quantum regimes that manifest below the Lamb-Dicke limit.

There are a number of basic quantum properties in a classical crystalline beam that may be exploited using standard measurement techniques; the spin states of the particles, the modes in the crystalline orbit, and the quantization in the emittance. These quantum properties cannot be controlled due to the high temperatures of the beams and therefore, are not useful for computational purposes. An ultracold crystalline beam is required in order to access quantum properties that are useful for computation.

Ion trap systems exploit two quantum properties of the ions in the trap, external eigenstates, such as the axial center-of-mass motion of the string of ions in the trap, and the internal eigenstates of each ion in the string. When sufficiently cooled, the string of ions in the trap has properties that can be used to define a set of computational basis states that can be operated on using laser excitations [10].

A significant challenge in quantum computing is controlling quantum decoherence $[16,17]$. However, research in ion traps has shown that quantum states in trapped ions can persist for very long times, even on the scale of minutes [15]. In a storage ring environment we will have to be sure to eliminate any sources of noise and other forms of energy that may disrupt the trapped ion quantum states. While we see this as an interesting problem, it falls beyond the scope of this paper. A good discussion of this topic is covered by Wineland et al. [15]. The scaling of the number of qubits $(N)$, while limited mostly to internal interactions, is related to the problem of decoherence. It has a unique temporal component we must consider, since not all ions in the crystalline beam can be operated on simultaneously. We will touch on this topic a little more in Sec. VI.

The major problems seen in ion traps remain concerns for a crystalline beam in a storage ring; spontaneous transitions in the vibrational motion, thermal radiation, and instabilities in various systems (i.e., laser power, rf voltages, and mechanical vibrations).

Inside the storage ring system, groups of smaller numbers of ions can be isolated from each other, using longitudinal rf potentials or by separating using velocity modulation in the cooling systems, creating isolated sets of qubits that can be operated on independently. The idea of such a multiplex environment, for ion traps, was discussed by Kielpinski $[18,19]$. In the storage ring environment, there is potential for multiplexing as well as an ability to work on ions and groups of ions in parallel. A storage ring could contain thousands of these smaller individual crystals. The many small chains of ions could serve different purposes, depending on the algorithm being employed. For example, some ion chains could be used as quantum memory and some could be used for other purposes, such as for systematic analysis. Having many ions and ion chains available opens up many possibilities. In addition, the length of the ion chains is not limited by the scale of the device. A chain could even wrap around and have a length that is longer than one turn of the accelerator. While this does not seem practical at this time, it illustrates the degrees of freedom such a structure will allow. What we find interesting is that having the ability to manipulate larger numbers of ions and groups of ions opens up interesting new questions. For example, can we entangle two independent groups of ions? Can we create a long chain of entangled ions and then split the chain, maintaining the entanglement? Can we reverse that process, take two shorter chains and connect them? Such manipulations require control over the axial potentials used to seperate the ions and surely raise many questions. But before we can solve a problem, we need to raise the question and imagine possibilities. Our discussion, for the rest of the manuscript, will not delve any deeper into these questions. They remain areas of research we hope to report on in the future.

There are other topics related to this work that are beyond the scope of this paper. While we discuss the use of various ions, our choices of ions, such as ${ }^{24} \mathrm{Mg}^{+}$and ${ }^{7} \mathrm{Li}^{+}$, are only for illustrative purposes. It is not our intent to discuss in this work the suitability of any particular ion for quantum computing. We take our guidance on what is suitable, to date, from current research in ion traps, but we do plan to look more deeply into this topic in the future. From the storage ring perspective, the choice of ion and ion velocity is a design constraint, only. We also do not go into the details of how to cool the ions to the sufficient degree required for quantum computing. We assume that what has been achieved in ion traps will be a guide to future research and take current ion trap research as proof that the required conditions are possible, for given ions. We understand we need to prove that we can achieve these conditions in a storage ring environment. This is, in fact, one intent of this paper, to put forward a concept that will motivate and start a discussion on methods to cool beams into the micro-Kelvin regime.

While we briefly discuss other storage ring approaches, our main focus is on circular radio frequency quadrupoles [20] (CRFQ) using nonrelativisitic, singly positive charged ions. This leads to many questions and potential issues, but we assume low intensity beams only, ignoring any space charge effects, and do not consider any other interactions, 
such as beam-gas, electron capture, or dissruption from thermal photons. All of these topics are discussed in other papers $[9,21]$ and while they create constraints on the parameter space, we prefer to keep the discussion focused on a basic design that could act as a starting point for further analysis.

Finally, we intentionally are trying to keep our discussion as simple as possible. We do not consider complex ion chains, but only describe linear, one dimensional chains of ions. We do not consider nonlinear optical effects or coupling. We do not discuss ion loss mechanisms and methods for reloading or replacing lost ions. For simplicity we assume the storage ring is filled to some well defined extent and that fill lasts long enough to be useful for a practical amount of computational work.

\section{RELATED WORK}

In the field of accelerators and beams, the concept of building a quantum computer using a particle beam storage ring is novel and has never been attempted. There has been extensive study of quantum effects in particle beam dynamics, which we will describe in the next sections. The creation of crystalline beams has been studied since the 1980s. Such beams represent a new state of matter that has not been extensively studied experimentally.

In the field of quantum computing, there are many technologies being studied as platforms for quantum computing, each of which have their own challenges and limitations. It is generally expected that a practical quantum computer be something that is scalable, have means for initializing qubits, allow operations within decoherence times, have methods for a universal set of operations, and allow qubits to be easily read [22].

It is worth noting that in the field of quantum computing, increasing the number of qubits is not necessarily going to lead to greater computational efficiency or linear scaling. This is especially true if one is fighting decoherence or looking to find ways to "hold" quantum states (e.g., quantum cavity). The number of qubits defines the dimensionality in a Hilbert space representing all the possible states in the quantum system. How to manage the computational efficiency with large $N$ needs to be understood.

Quantum computers that might be compared to a storage ring system would be those that attempt to operate at the atomic level. A trapped ion quantum computer will confine ions or other charged particles in some free space using electromagnetic fields $[10,23]$. The qubits are the quantum states associated with each ion. Ion trap systems are scalable, in principle, and hold much promise as quantum computing systems. A storage ring system is very similar to an ion trap. A significant difference is the particles will be confined into a circular electromagnetic guide. Storage ring ions will have finite average velocities, although velocity is a free parameter that can be optimized. Ions in a storage ring can be entangled, as they can in an ion trap. Another type of system is nuclear magnet resonance (NMR) quantum computing [24,25], which has been explored experimentally and is the first system to demonstrate Shor's algorithm [26,27]. However, NMR systems have not been shown to scale beyond a few qubits [28]. A recent survey of quantum computing technologies was explored by Gyongyosi and Imre [29].

A number of groups are researching ways to scale and overcome the difficulties of working with and controlling a large number of ions. In our work we propose the idea of placing chains of ions into individual bunches, separated, for example, by some rf potential. Some groups are looking at ways of managing despite the problems with controlling a large number of ions. For example, work has been done in understanding transverse vibrational states as opposed to using longitudinal vibrational states to enable quantum gate operations [30].

\section{CLASSICAL CRYSTALLINE BEAMS}

A classical crystalline beam is formed by cooling [31] the beam sufficiently to a point where the charged particles "lock into" a structure in which repelling Coulomb forces balance against external forces. This transforms the basic ideal gas particle beam distribution into a new form of matter [32,33], a chain of particles locked into a sequence in much the way atoms get locked into a lattice in a crystal. These types of beams have been extensively studied theoretically $[34,35]$ and to some extent experimentally [36-38].

The separation of the particles in a crystal scales on the order of $\nu_{\text {eff }}^{-2 / 3} \xi$, where $\xi$ is called the characteristic distance,

$$
\xi=\left(q^{2} r_{0} \rho^{2} / A \beta^{2} \gamma^{2}\right)^{1 / 3},
$$

where $q$ is the charge, $A$ is the atomic number, $r_{0}$ is the classical particle radius, $\rho$ is the average bending radius in the storage ring lattice, $\beta, \gamma$ are the relativistic factors, and

$$
\nu_{\text {eff }}^{2}=\min \left(\nu_{y}^{2}, \nu_{x}^{2}-\gamma^{2}\right),
$$

where $\nu_{x}$ and $\nu_{y}$ are the betatron oscillations resulting from the focusing structure of the accelerator, in the horizontal and vertical planes, respectively. Note that this scaling is just a rough estimation of the distance between the ions of the crystal. A more precise description of the crystal structure will be discussed later, using the Wigner-Seitz radius, which defines the density of the crystal based on the secular motion of the ions in the crystal.

\section{ION COULOMB CRYSTALS}

To control the state of the ions in a classical crystalline beam would be difficult, since the standard methods, developed for ion traps, require the vibration states of the ions to be very small. 
The de Broglie wavelength of a high energy particle is

$$
\lambda_{d b}=\hbar / p_{\perp} \leq \lambda_{c} \sqrt{\frac{\beta}{\gamma \epsilon_{n}}},
$$

where $\lambda_{c}=\hbar c / m c^{2}$ is the Compton wavelength. The de Broglie wavelength, in the beam rest frame, when equal to the highest phonon frequency, $\max \left(\omega_{\mathrm{ph}}\right)=\sqrt{2} \gamma \omega_{\beta}$ marks the point in which external quantum modes become observable and vibration states become small enough that internal quantum states can be manipulated.

$$
\frac{\hbar}{2} \max \left(\omega_{\mathrm{ph}}\right) \approx \frac{k_{B} T}{2} .
$$

The beam cooling systems, to get beams to this state, will need to bring the temperature down by as much as an additional factor of $10^{4}$ (e.g., from $100 \mathrm{mK}$ to $10 \mu \mathrm{K}$.) To cool beams down to this level may require additional systems that remove all sources of noise that can add heat to the beams, such as cryogenically cooled beam pipes and vibration compensation systems. Most importantly, though, the lattice structure of the accelerator will need to be carefully designed to reduce large perturbations that may disrupt the crystalline beams or make it too difficult to reach the right conditions for creating ion Coulomb crystals.

An ion Coulomb crystal can be described, in a classical sense, as a string of charged masses acting as simple harmonic oscillators. Such a structure can be described in one dimension as masses coupled by springs, where the motion is small compared to the distances between the ions and so the spring coefficient, $m \omega_{0}^{2}$, is taken as constant. Here, $\omega_{0}$ is the fundamental frequency for the chain of ions and is a function of the Coulomb and other potentials holding the ions in the chain. In this case, the motion is seen as modes in the axial motion of the ions. Looking at it in three dimensions [30], where we have $\mathrm{N}$ independent harmonic oscillators each of charge +1 and mass $m$, the Hamiltonian can be expressed as

$H=\sum_{u} \sum_{j=1}^{N} \frac{p_{u, j}^{2}}{2 m}+V, \quad$ where $V=V_{\text {trap }}+V_{\text {Coulomb }}$

where $u=\{x, y, z\}$. The masses and charges of each ion are identical. The potential is the sum of applied voltages and the Coulomb potential of the adjacent ions, or,

$$
V=\frac{m}{2} \sum_{j=1}^{N}\left(\omega_{x}^{2} x_{j}^{2}+\omega_{y}^{2} y_{j}^{2}+\omega_{z}^{2} z_{j}^{2}\right)+\sum_{n, j} \frac{e^{2}}{4 \pi \epsilon_{0} r_{n, j}}
$$

where $r_{n, j}$ is the distance between ions $n$ and $j$ and $\omega_{u}$ is the center of mass frequency (of the system) along the $u$ direction. We define $d$ to represent a unitless scale length, $d_{n}=z_{n}^{0} / l$, where $l^{3}=e^{2} /\left(4 \pi \epsilon_{0} m \omega_{z}^{2}\right)$. The potential can be expanded out as a Taylor series, where the first order is zero (by definition) and the second order represents a harmonic approximation of the potential seen by the ions.

$$
V=\frac{1}{2} \sum_{\xi, n, j} q_{n}^{\xi} q_{j}^{\xi}\left[\frac{\partial^{2} V}{\partial \xi_{n} \partial \xi_{j}}\right]_{\xi_{n}=\xi_{n}^{0}}
$$

where we are expanding around the ion equilibrium positions, $\xi_{n}^{0}=\left(x_{n}^{0}, y_{n}^{0}, z_{n}^{0}\right)$, such that $\xi_{n}=\xi_{n}^{0}+q_{n}^{\xi}, q_{n}^{\xi}$ representing the position of ion $n$ in the direction $\xi$. The potential can now be written as,

$$
V=\frac{m \omega_{z}^{2}}{2} \sum_{\xi, n, j} A_{n, j}^{\xi} q_{n}^{\xi} q_{j}^{\xi}
$$

where,

$$
\begin{gathered}
A_{n, j}^{\xi}=\beta_{\xi}^{2}+\sum_{p=1, p \neq j}^{N} \frac{a_{\xi}}{\left|d_{j}-d_{p}\right|^{3}} \quad \text { for }(n=j) \\
A_{n, j}^{\xi}=\frac{-a_{\xi}}{\left|d_{j}-d_{n}\right|^{3}} \quad \text { for }(n \neq j)
\end{gathered}
$$

and,

$$
\beta_{\xi}=\frac{\omega_{\xi}}{\omega_{z}}, \quad a_{x}=a_{y}=-1, \quad \text { and } \quad a_{z}=2
$$

the eigenfrequencies are

$$
\omega_{\xi, k} \equiv \sqrt{\lambda_{\xi, k}} \omega_{\xi}
$$

and eigenvectors $b_{j}^{\xi, k}$ are obtained from diagonalization of $A^{\xi}=\left[A_{n, j}^{\xi}\right]$. Then,

$$
\sum_{n} A_{n, j}^{\xi} b_{n}^{\xi, k}=\lambda_{\xi, k} b_{j}^{\xi, k}
$$

substituting,

$$
q_{j}^{\xi}(t)=\sum_{k} b_{j}^{\xi, k} \sqrt{\frac{\hbar}{2 m \omega_{\xi, k}}}\left(a_{\xi, k}+a_{\xi, k}^{\dagger}\right)
$$

then,

$$
H_{0}=\sum_{j=1}^{N} \hbar \omega_{j}\left(a_{j}^{\dagger} a_{j}+\frac{1}{2}\right),
$$

where the annihilation and creation operators for the $j$ th phonon mode, $a_{j}^{\dagger}$ and $a_{j}$, are a function of the mass and 
charge of the ions and the potential constraining the ions in the chain. In this case, the quantized vibrational energy per mode $n$, is

$$
E_{n}=\left\langle n\left|H_{0}\right| n\right\rangle=\left(n+\frac{1}{2}\right) \hbar \omega_{0} .
$$

These phonon modes are extremely important in establishing a quantum gate. The ions in the chain form individual qubits, and the quantized phonon modes provide an additional quantum bit common to all the ions, and thus serves a special purpose for quantum logic operations that can be used to entangle the qubits. This is discussed further in Sec. VID.

What is clear is that as the number of ions increase, the number of modes also increases. An ensemble of ions of size $N+1$ has $3 N+3$ motional degrees of freedom, so as $N$ become large, cooling and controlling the chain becomes very difficult. However, in a storage ring, we can imagine isolating two small ensemble of ions, cooling them independently, and then adiabatically merging the two chains, each already in a ground state. This illustrates one of the ways a storage ring system could build up larger chains of ions.

\section{EXAMPLE OF CRYSTALLINE ION BEAMS}

A practical example of a storage ring for crystalline beams was explored using the PAul Laser cooLing Accelerator System (PALLAS) at LMU Munich [37-39].

In the PALLAS experiments, they observed ${ }^{24} \mathrm{Mg}^{+}$ion beams with $\beta c \approx 10^{-5} \mathrm{c}$ transition into a crystalline state for both coasting beams and bunched beams [40]. The issues in controlling and maintaining the crystalline structure [41] were related to the modulation of the beam envelope due to the AG fields and velocity dependent shear forces. They observed that the coupling driven by the transverse motion into thermal motion set an upper limit to the confinement strength. This limit was observed to be a factor of three greater than the criterion that the lattice periodicity be greater than $2 \sqrt{2} \nu_{\text {eff }}$. For their choice of ions and rf parameters, the ratio of the periodicity to the tune was over 11 and independent of the beam velocity (although, as will be seen below, both the periodicity and tune are each velocity dependent.)

An interesting observation is the effect of velocity dependent shear forces on crystal formation. In principle, if we could achieve the same normalized phase space for higher velocity ions that were achieved for lower velocity ions, then the temperature of the beam should be constant. Velocity dependent shear is a unique feature of a CRFQ structure, since the particles at low velocity are well confined by the transverse $\mathrm{rf}$ field. Intuitively, as the velocity increases, off momentum particles will have larger deviations from the design orbit if the rf frequency and voltage are fixed. This can be expressed quantitatively as a velocity dependence on the dispersion. Even for a constant temperature beam, the increase in dispersion means there will be larger orbit deviations for off momentum particles.

Ion trap systems work within a regime in which centerof-mass vibrational degrees of freedom (phonon states) can be operated on as a computational basis of states [10]. Ion trap systems also slow ions down to a point in which each ion is vibrating in a fixed spatial region in the chain of ions in the trap [15]. For a storage ring system, we would establish a similar state, but in a rotating frame. The crystal would rotate at a fixed frequency with all ions vibrating within a small region of space in that frame. This means the spacing of the ions would be fixed and stable, to a level that a set of laser systems, for example, could excite internal ion states and external crystal states.

PALLAS and S-LSR [42] were two facilities that have studied crystalline beams and explored what conditions are best for creating this state of matter for those given lattices. They did not explore ultracold Coulomb crystalline beams.

The PALLAS ring was a CRFQ (circular radio frequency quadrupole) ring [43]. In the following, we will mostly follow the formalism adopted by Schramm and Habs [21].

The azimuthal motion of the ions is an angular precession at the frequency,

$$
\omega_{0}=\beta c / R,
$$

where $\mathrm{R}$ is the radius of curvature of the ring. The alternating focusing period is $L=\beta \lambda$, where $\lambda=c / f_{\text {rf }}$ is the rf field wavelength and $\beta$ is the relativistic factor. Transverse motion in a rf quadrupole ring, when the bending radius is much larger than the aperture, can be described using the equations of motion for a linear $\mathrm{rf}$ quadrupole trap, where the periodicity is a function of the velocity,

$$
P=\frac{C}{L}
$$

where $C=2 \pi R$ and $L=v_{0} / f_{\mathrm{rf}}$. The rf quadrupole configuration has an electric potential,

$$
\Phi(\hat{x}, \hat{y}, \hat{z})=\frac{\Phi_{0}(t)}{2 r_{0}}\left(\hat{x}^{2}-\hat{y}^{2}\right),
$$

where an alternating potential is applied as,

$$
\Phi_{0}(t)=U_{d c}-U_{\mathrm{rf}} \cos (\Omega t),
$$

where $U_{\mathrm{rf}}$ is the rf voltage and $\Omega$ is the rf angular frequency. The equations of motion can be written in canonical form of a linear differential equation with periodic, variable coefficients (Mathieu's differential equation) [21], 


$$
\frac{d^{2} u}{d \xi^{2}}+\left[b_{u}-2 q_{u} \cos (2 \xi)\right] u=0
$$

where,

$$
\begin{aligned}
& b_{u}=b_{\hat{x}}=-b_{\hat{y}}=\frac{4 e U_{d c}}{m \Omega^{2} r_{0}^{2}}, \\
& q_{u}=q_{\hat{x}}=-q_{\hat{y}}=\frac{2 e U_{\mathrm{rf}}}{m \Omega^{2} r_{0}^{2}},
\end{aligned}
$$

and,

$$
\xi=\frac{1}{2} \Omega t
$$

We will return to the value of $q$, which represents the ratio of the potential to the kinetic energy of the driven motion. The stable motion in the transverse directions can be described by the secular frequency,

$$
\omega_{\mathrm{sec}, u}=\beta_{u} \Omega / 2 .
$$

The value of $\beta_{u}$ depends on the trap parameters, $q_{u}, b_{u}$, where we choose to be in a region of weak confinement $(q \ll 1$ and $b \leq q)$, where only the first term in the series of harmonics of the driving frequency dominates, so

$$
\omega_{\mathrm{sec}} \approx q \Omega / \sqrt{8} .
$$

For axial confinement in a storage ring, a potential is applied to ring electrodes or by using biased sections at the ends of the quadrupole electrodes,

$$
\Phi_{z}(r, z)=\frac{\kappa U_{\text {end }}}{r_{0}^{2}}\left(z^{2}-\frac{r^{2}}{2}\right)
$$

where $\kappa$ is a constant based on the geometry.

Given this description of a CRFQ storage ring, simple parameters can be defined that describe the general motion of the ions. The phase advance per cell, in this structure, is

$$
\mu=\int_{z}^{z+L} \frac{1}{\omega(s)} d s=\frac{L}{\bar{\omega}^{2}}=2 \pi \frac{L}{\lambda_{\beta}},
$$

where a smooth approximation is assumed in which the variation of the beam envelope over one focusing cell is small and the amplitude function, $\omega(z)$ can be approximated by a mean value $\bar{\omega}$ and a small modulation (the micromotion in the $\mathrm{rf}$ driven system). The micromotion corresponds to an oscillation with a short wavelength of the focusing section,

$$
L=\beta \lambda=2 \pi v_{0} / \Omega
$$

where the secular motion is defined as,

$$
\omega_{\mathrm{sec}} \equiv \omega_{\beta}=2 \pi v_{0} / \lambda_{\beta} .
$$

$\lambda_{\beta}$ is the betatron wavelength. The beta-function and dispersion function can be exactly replaced by their mean values, for $\lambda_{\beta} \gg L$. For a storage ring rf quadrupole, there is a set of simple relations for describing the lattice structure [21].

$$
\text { Periodicity } P=C / L
$$

$$
\text { Tune } Q=C / \lambda_{\beta}=\omega_{\mathrm{sec}} C / 2 \pi v_{0}
$$

Beta-function $\quad \beta_{0}=R / Q[\mathrm{~m}]$

$$
\text { phase } \phi(z)=Q z / R
$$

$$
\text { dispersion } \quad D_{0}=R / Q^{2}[\mathrm{~m}]
$$

momentum compaction $\quad \alpha=D_{0} / R=1 / Q^{2}$

$$
\text { transition energy } \quad \gamma_{t r}=Q
$$

The PALLAS ring was a "table-top" size accelerator, with a radius, $R_{0}=57.5 \mathrm{~mm}$. For ${ }^{24} \mathrm{Mg}^{+}$beams with velocities $v_{0}=1000-2500[\mathrm{~m} / \mathrm{s}]$, it had cell lengths, $L=0.16-0.4[\mathrm{~mm}]$, respectively, and periodicities, $P=2272-910$, respectively. In this range the tunes are on the order of $Q \approx 60$ and dispersion $D_{0} \approx 1.6 \times 10^{-4}[\mathrm{~m}]$. The periodicity was well above the criterion of $P>$ $2 \sqrt{2} \nu_{\text {eff }}$ for the formation of crystalline beams. The CRFQ ring leads to a highly symmetric lattice with smooth field variations and, experimentally, was effective in creating crystalline beams. However, they were not able to store beams below $v_{0}=800[\mathrm{~m} / \mathrm{s}]$. This was due to residual modulation of the potential seen by the ions in the ring. Such small potentials could come from small traps in the vacuum chamber (a change in impedance) or charge buildup on viewports. The scheme for acceleration depended on ions remaining trapped in rf potential wells while initial interaction with the laser beam was performed. Ion velocities were controlled using the counterpropagating laser beam kept at a fixed frequency.

The S-LSR ring was a geometric AG storage ring with a circumference of $22.557 \mathrm{~m}$. It was built for the purpose of studying methods of cooling ion beams. Such a structure suffers from "shear heating," in which momentum dispersion in the bending magnets causes angular velocity differences among the ions with different energies. The SLSR group studied this problem in great detail. Two approaches can be used to suppress the shear. A tapered cooling system using a Wien filter will enable cooling to take place in a lattice with finite dispersion. Another approach is to adopt a lattice with no linear dispersion using a dispersion suppression system. Such a system creates a cross-field of magnetic and electric fields. An electric field from a cylindrical electrostatic deflector is superimposed with the magnetic field, enhancing the radial 
focusing. By adjusting the focusing, the linear dispersion can be suppressed throughout the lattice.

\section{A STORAGE RING QUANTUM COMPUTER}

Cooling a beam in a storage ring into the quantum regime has never been done. Most systems have studied the cooling of beams with fairly high velocities. Ion traps cool beams to stationary steady states.

One important condition is to ensure that for the ensemble of ions the ratio of the energy of the mutual Coulomb repulsion to the thermal energy is high enough that we can establish the condition of Eq. (8). If we express this using the unitless plasma parameter [21],

$$
\Lambda_{p}=\frac{1}{4 \pi \epsilon_{0}} \frac{e^{2}}{a_{\mathrm{ws}} k_{B} T}
$$

where, $a_{\mathrm{ws}}$ is the Wigner-Seitz radius,

$$
a_{\mathrm{ws}}=\left[\frac{1}{4 \pi \epsilon_{0}} \frac{3 e^{2}}{2 m \omega_{\mathrm{sec}}^{2}}\right]^{1 / 3},
$$

and $\omega_{\text {sec }}$ is the secular frequency of the beam, given by

$$
\omega_{\mathrm{sec}}=\frac{2 e U_{\mathrm{rf}}}{\sqrt{8} m \Omega r_{0}^{2}},
$$

then we can see that, in addition to cooling to a low temperature, this suggests we use a beam with lower mass ions at a high rf potential, to make $\Lambda_{p}$ as large as possible.

To create the ion Coulomb crystal, the temperature needs to be lowered to the point that thermal vibrations are small compared to the quantum phonon modes for the crystal. If we treat the crystal as just a chain of ions with equilibrium spacings of $a$, the only force the ions see from each other is from the Coulomb potential of the neighboring ions. The potential seen by two adjacent ions separated by $10 \mu \mathrm{m}$ is on the order of $3 \times 10^{-4} \mathrm{eV}$. This Coulomb potential energy is what determines the spring constant, $K=\left(d^{2} U / d r^{2}\right)_{r=a}=m \omega_{0}^{2}$, between the ions that determines the axial phonon modes for the crystal. In a classical treatment, the angular frequency for the phonon modes is,

$$
\omega_{\mathrm{ph}}=\sqrt{\frac{4 K}{m}} \sin (k a / 2) .
$$

Here, $k$ is the wave number $k=2 \pi / \lambda$, and $\omega_{\mathrm{ph}}$ is a periodic function of $k$, symmetric with respect to $k$ and $-k$ with its first period between $k=-\pi / a$ and $k=\pi / a$. The maximum frequency will correspond to when $\sin (k a / 2)=1$.

$$
\max \left(\omega_{\mathrm{ph}}\right)=\sqrt{\frac{4 K}{m}}
$$

If we use the Coulomb potential, with ion spacings of $10 \mu \mathrm{m}$, we see that for the ${ }^{24} \mathrm{Mg}^{+}$crystalline beam, $\max \left(\omega_{\mathrm{ph}}\right) \approx 3 \mathrm{MHz}$.

It is important to note that there remains a dispersion to the spatial proximity of each ion in the crystal [10],

$$
\Delta z_{\mathrm{cm}}=\sqrt{\hbar / 2 N m \omega_{\mathrm{ph}}} .
$$

Returning to Eq. (8), we know that temperature is directly proportional to the momentum spread in the beam and the average kinetic energy of the beam. We now have an estimate for what temperature is needed in order to create an ion Coulomb crystal, given the value of $\max \left(\omega_{\mathrm{ph}}\right)$ for a beam with $a=10 \mu \mathrm{m}$. The corresponding temperature is on the order of $2.3 \times 10^{-5} \mathrm{~K}$. This sets the scale for the range of values for $\delta p / p_{0}$ and the velocity of the ions. From Eq. (3), we see that the product $\left(\frac{\delta p}{p_{0}}\right) E_{0}=2.3 \times 10^{-5} / 2.32 \times 10^{4} \approx 1 \times 10^{-9}$. A beam with $E_{0}=1[\mathrm{eV}]$, corresponding to a velocity of $2818 \mathrm{~m} / \mathrm{s}$, would have to be cooled to have a $\delta p / p_{0}=1 \times 10^{-9}$, which is very challenging [44]. However, a beam with $E_{0}=10^{-4}[\mathrm{eV}]$, with a velocity near $28 \mathrm{~m} / \mathrm{s}$, would have to be cooled to have a $\delta p / p_{0}=1 \times 10^{-5}$. So, making the beam energy as low as possible seems to improve the ability to reach the low temperatures needed to make an ion Coulomb crystal. We do not mean to overemphasis the importance of the ion's kinetic energy in relation to cooling. Our simple observation is that in ion traps, where ions are stationary, very low temperatures have been achieved, while for higher energy ions, such as in the PALLAS experiments, success was limited to some range of ion velocities. We do not think this is a fundamental limitation, but a result of choices in a complex parameter space.

In Table I, we have gone through a simple exercise, to try and understand how to develop parameters that will create an ion Coulomb crystal. The assumption is we can cool the beams to a level where the $\delta p / p$ is $10^{-7}$. For this exercise we recalculate the parameters for a PALLAS-like ring to

TABLE I. Parameters needed to create an ion Coulomb crystal in a circular rf quadrupole for three different types of ions, where we assume cooling has achieved $\delta p / p=10^{-7}$, all with a $C=1 \mathrm{~m}$ ring.

\begin{tabular}{lcccc}
\hline \hline Parameter & ${ }^{24} \mathrm{Mg}^{+}$ & ${ }^{9} \mathrm{Be}^{+}$ & ${ }^{7} \mathrm{Li}^{+}$ & Units \\
\hline$v_{\max }$ & 276 & 580 & 700 & $\mathrm{~m} / \mathrm{sec}$ \\
$f_{\text {rf }}$ & 6.3 & 12.6 & 12.6 & $\mathrm{MHz}$ \\
$L$ & 43 & 46 & 56 & $\mu \mathrm{m}$ \\
$P$ & 22826 & 21650 & 17950 & \\
$Q$ & 1963 & 1257 & 1340 & \\
$D_{0}$ & $4.1 \times 10^{-8}$ & $1 . \times 10^{-7}$ & $8.9 \times 10^{-8}$ & $\mathrm{~m}$ \\
$T_{\max }$ & 22 & 37 & 42 & $\mu \mathrm{K}$ \\
$\max \left(\omega_{\mathrm{ph}}\right)$ & 3. & 4.8 & 5.4 & $\mathrm{MHz}$ \\
$\Lambda_{p}$ & 83140 & 44290 & 42560 & \\
$\Delta z_{\mathrm{cm}, \max }$ & 5.7 & 7.3 & 7.6 & $\mathrm{~nm}$ \\
\hline \hline
\end{tabular}


have a circumference of $1 \mathrm{~m}$. There were two basic constraints we applied; that the value of $P / Q$ be greater than 8.5 and the achieved temperature would be equal to that corresponding to the maximum phonon frequency. This maximum temperature we correlate to a maximum velocity of the beams, although we note that this is just as a point of reference. We also note that by increasing the circumference of the ring from $0.36 \mathrm{~m}$ to $1 \mathrm{~m}$, we reduced the average dispersion by $1 / 0.36$. We have already seen that the dispersion is velocity dependent, and this can constrain the ability to create crystals due to the larger orbital excursions of off momentum particles.

As a matter of reference, PALLAS experiments were able to cool beams down to the Doppler limit (for their parameter space), to a temperature of $<3 \mathrm{mK}$ and achieved a $\Lambda_{p} \approx 500$.

In Fig. 1 we look at the range of rf frequencies and voltages that must be considered to create the conditions for an ion Coulomb crystal, in the case of ${ }^{7} \mathrm{Li}^{+}$with $v_{0}=100 \mathrm{~m} / \mathrm{s}$, for the $C=1 \mathrm{~m}$ ring. Since we know from the PALLAS experience that $P / Q$ must be greater than 8.5 , we see that any set of (frequency, voltage) values must fall in the range above the solid black line.

In Fig. 2 we show the surface of values for the WignerSeitz radius, for the same range of frequencies and voltages, again for ${ }^{7} \mathrm{Li}^{+}$ions with $v_{0}=100 \mathrm{~m} / \mathrm{s}$. As a reference, a dashed line is shown where the radius is $10 \mu \mathrm{m}$ and a line is drawn showing where the corresponding values of $P / Q=8.5$. These are the first set of constraints that will apply to the design of the storage ring.

Another parameter that affects the crystalline structure is the amount of transverse spread in the motion of the ions.
This can be expressed as a focusing apparent plasma parameter,

$$
\Lambda_{\mathrm{app}, f} \propto \frac{P^{2}}{\lambda Q^{2}} .
$$

As with $\Lambda_{p}$, the larger this number the better the conditions are for an ion Coulomb crystal. So, this suggests a larger $f_{\text {rf }}$, larger $r_{0}$, and smaller $U_{\text {rf }}$ are preferred. There is an obvious trade-off required on finding the optimal rf voltage. But, as seen in Fig. 2, pushing voltage too low or frequency too high will result in larger values of the Wigner-Seitz radius (lower density ion Coulomb crystal).

In Fig. 3 we show the surface of values for the plasma parameter $\left(\log _{10} \Lambda_{p}\right.$ is what is plotted) as a function of momentum spread and rf voltage at an rf frequency of 12.6 Mhz. Drawn on the figure is the contour for $\Lambda_{p}=40,000$, above which is where ion Coulomb crystals useful for quantum computing would form. For the $v=$ $100 \mathrm{~m} / \mathrm{s}$ ions we can see the range of $\delta p / p$ must be less than $10^{-5}$. The lower the voltage the more cooling that is needed.

Finally, the PALLAS results showed that velocity dependent shear was what limited the formation of crystalline beams at higher velocities. Again, this can be expressed in terms of an apparent plasma parameter,

$$
\Lambda_{\text {app }, s} \propto \frac{Q^{2}}{\lambda} .
$$

This is quadratically dependent on velocity and the radius of the ring, improves quadratically with increasing $\mathrm{rf}$

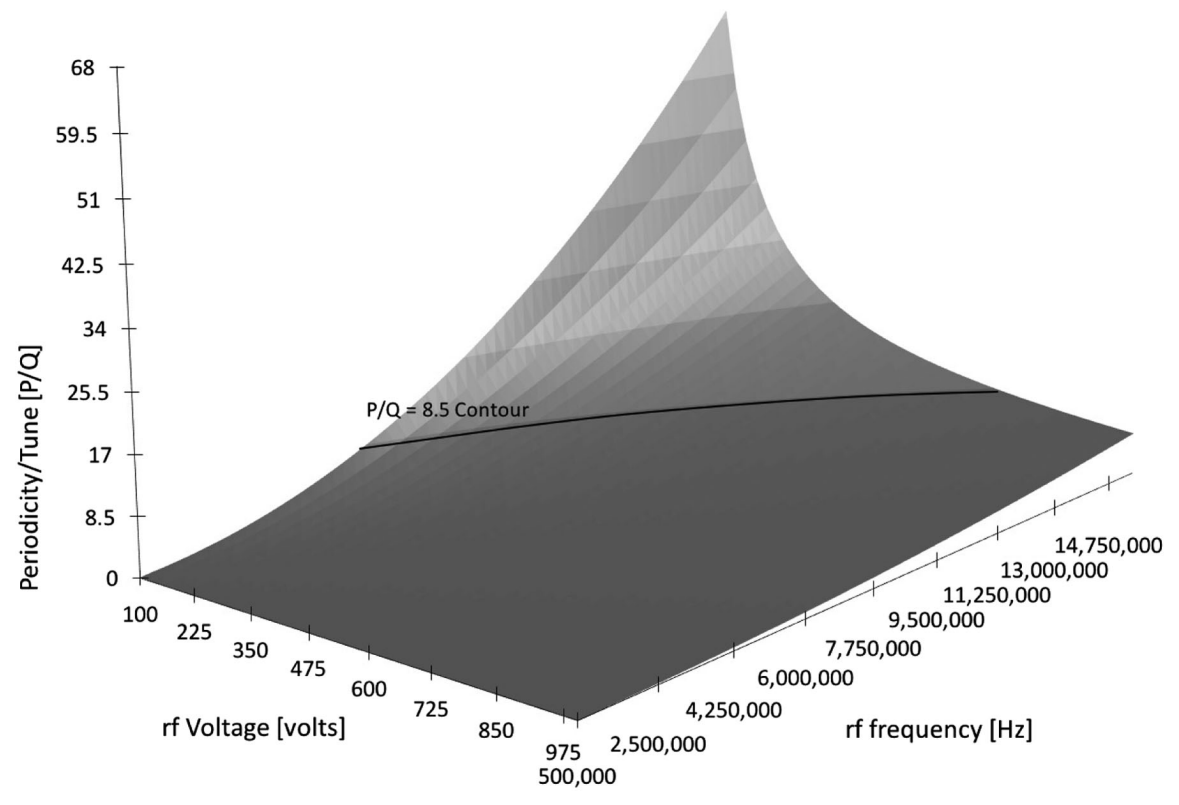

FIG. 1. The surface of values of $P / Q$ for a range of rf frequencies and voltage for ${ }^{7} \mathrm{Li}^{+}$ions with $v_{0}=100 \mathrm{~m} / \mathrm{s}, r_{0}=2.5 \mathrm{~mm}$, and $C=1 \mathrm{~m}$. 


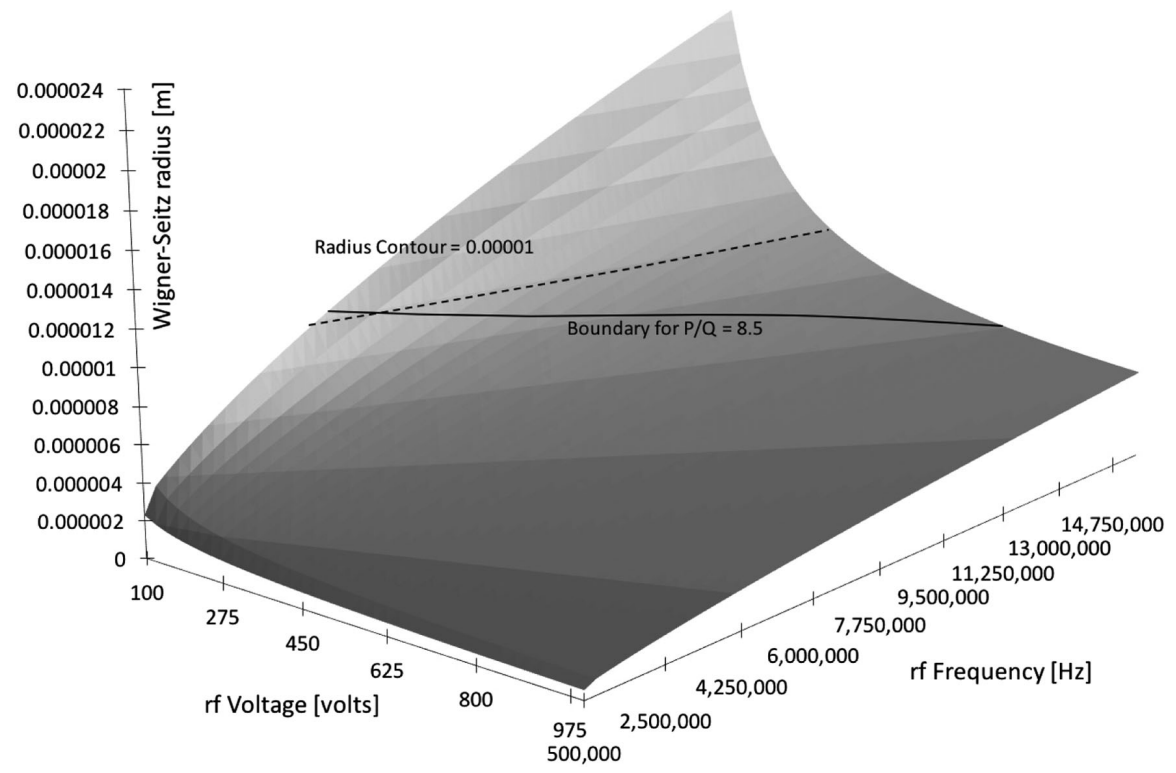

FIG. 2. The surface of values of Wigner-Seitz radii for a range of $\mathrm{rf}$ frequencies and voltage for ${ }^{7} \mathrm{Li}^{+}$ions with $v_{0}=100 \mathrm{~m} / \mathrm{s}$.

voltage and frequency, and improves with smaller aperture. We already explained how off momentum orbital excursions are a function of velocity dependent dispersion. Now we can see that maximizing these plasma parameters is basically a method for minimizing those excursions and we can see how this can be done for a given ion velocity.

In Fig. 4 we show the surface of values for the apparent plasma parameter $\left(\log _{10} \Lambda_{\text {app, } s}\right.$ is what is plotted) as a function of ion velocity and rf voltage, for the same parameters as in Fig. 3. For the ${ }^{7} \mathrm{Li}^{+}$parameters in Table I, the value of $\Lambda_{\text {app, } s}$ is about 75,000. For reference, a line was drawn on Fig. 4 showing where this value lands on the surface.
Overall, what the PALLAS experience suggests is that to get to the quantum regime for crystalline beams in a CRFQ structure, use of low mass ions, such as ${ }^{7} \mathrm{Li}^{+}$or ${ }^{9} \mathrm{Be}^{+}$, at low velocities (e.g., $1 \lesssim v \lesssim 100[\mathrm{~m} / \mathrm{sec}]$ ) may be important. The laser cooling will need to reduce the $\delta p / p$ to less than $10^{-5}$. However, these parameters need to be studied much more carefully, since many other factors play into the choice of ions and velocity. While the experimental evidence from PALLAS shows limitations in the range of velocities, more studies are needed to better understand these limits. We know that for higher velocities, we need to remain in the Mathieu's region of stability [21] (we simply run into limitations for a structure that uses only electric

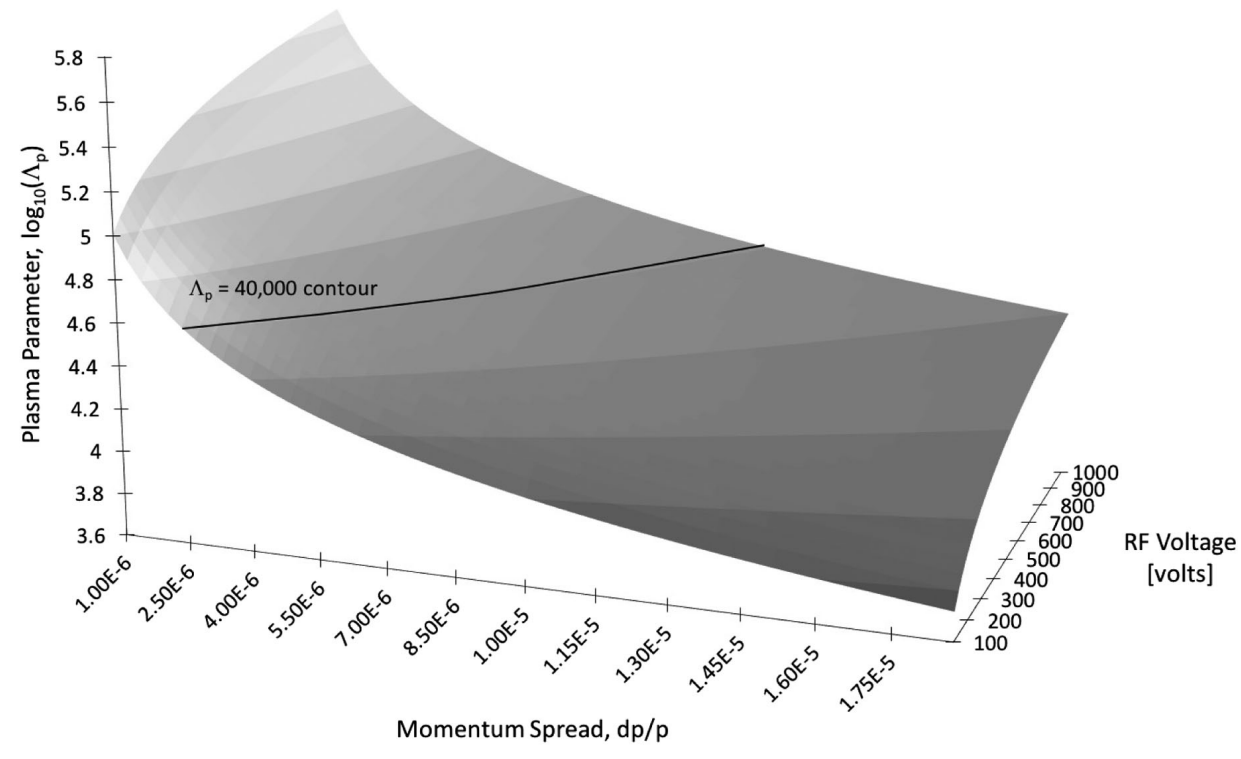

FIG. 3. The surface of values of the $\log _{10} \Lambda_{p}$ versus momentum spread and voltage for ${ }^{7} \mathrm{Li}^{+}$ions with $v_{0}=100 \mathrm{~m} / \mathrm{s}$, at $f_{\mathrm{rf}}=12.6 \mathrm{Mhz}$. 


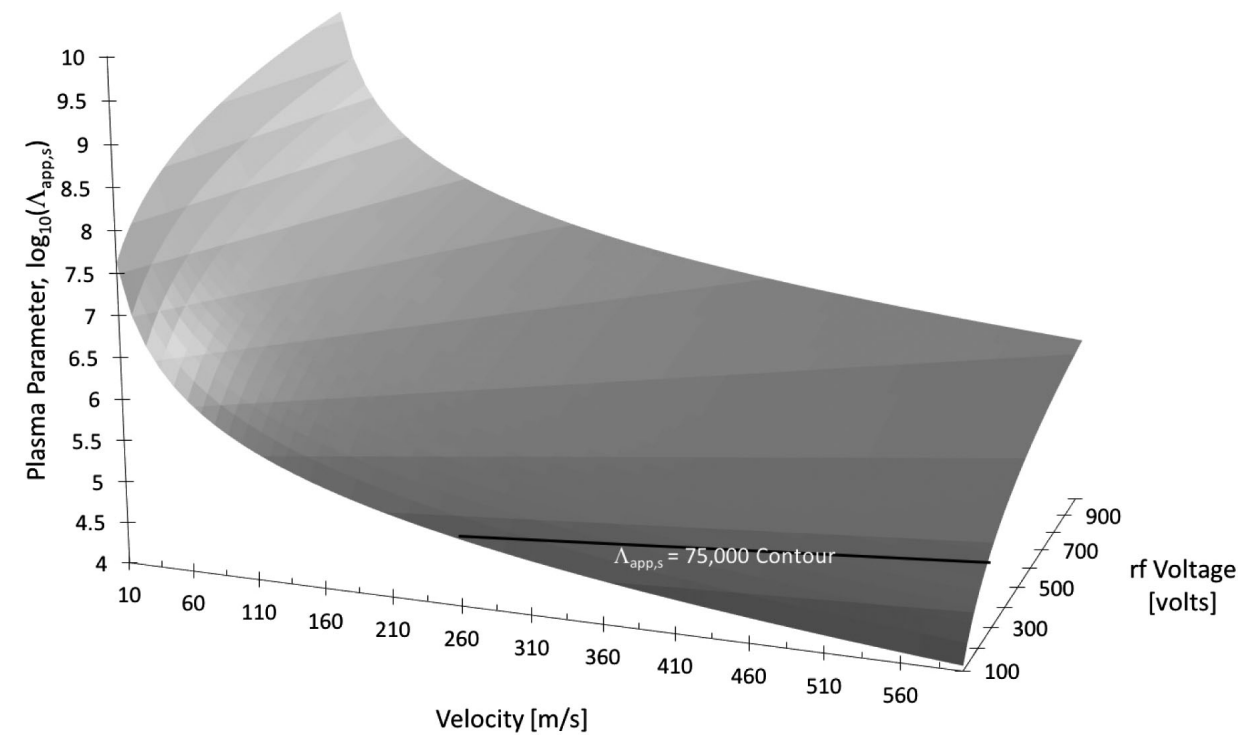

FIG. 4. The surface of values of the $\log _{10} \Lambda_{\text {app }, s}$ versus velocity and voltage for ${ }^{7} \mathrm{Li}^{+}$ions with $f_{\text {rf }}=12.6 \mathrm{Mhz}$.

fields.) But, even keeping the parameter space as simple as possible and assuming we can scale higher by employing magnetic bending (ignoring space charge, intrabeam scattering, and other effects), can we scale up in velocity while maintaining the crystal structure? At lower velocities, can we develop an extremely low impedance ring and find ways to prevent or control unwanted potentials from building?

The choice of ions involves consideration of the laser frequencies and power, coherence times for given excitation states, and other considerations. So while ${ }^{7} \mathrm{Li}^{+}$is a desirable choice from the point of view of its low mass, the excitation states are not in a convenient range, making it a less than optimal choice.

Experience with ion traps has shown that achieving a maximum switching rate for quantum computing, which is limited by the frequency and duration of the laser pulses, carefully configured to rotate an ions internal state without affecting the center-of-mass motional state, places further constraints on the system. Consider that the extent of the ground-state vibrational wave function, $\Delta z_{\mathrm{cm}}$, is limited by the number of ions being acted upon, or,

$$
\Delta z_{\mathrm{cm}} k_{z}=\left(\frac{\hbar k^{2} \cos ^{2}(\theta)}{2 N m \omega_{z}}\right)^{1 / 2} \equiv \frac{\eta}{\sqrt{N}}
$$

Here, $k_{z}=k \cos (\theta)$ is the wave vector component along the $z$ direction in the trap and $\eta$ is the Lamb-Dicke parameter for a single trapped ion. This can be expressed in terms of the energy of the recoil of an ion after emission of a single photon,

$$
\eta=\cos (\theta)\left(E_{R} / \hbar \omega_{z}\right)^{1 / 2},
$$

where $E_{R} \equiv(\hbar k)^{2} / 2 m$. The value for $\eta$ is a function of the vibrational states (through $\omega_{z}$ ) for the harmonic motion of the trapped ion. Since the ions are trapped in both radial and axial directions, there is a Lamb-Dicke parameter for radial confinement and a parameter for axial confinement. Radial confinement in a trap is stable as long as there is a balance between the potential and kinetic energy of the driven motion ( $q$ from Eq. (27) is less than 1). Radial micromotion has a velocity amplitude that is a function of this ratio and the distance particles deviate from the central axis. The Lamb-Dicke parameter for radial motion, is

$$
\eta_{r}=\left(\frac{2 \sqrt{2} E_{R} k^{2} r_{0}^{2}}{e U_{\mathrm{rf}}}\right)^{1 / 4}
$$

To establish a stable chain of ions, the radial confinement must be stronger than the axial confinement, requiring $\eta_{r}$ to be much smaller than 1. Likewise, axial confinement must be balanced and is controlled with a longitudinal potential.

\section{A. Detailed considerations}

From a practical perspective, the storage ring needs a location for injection of the ion beam, locations for beam cooling, locations to read and write the qubits, locations for diagnostic instrumentation, and locations to apply rf for axial confinement.

To achieve ultracold crystalline beams, we want to avoid large sudden transients in the magnetic field lattice, as the ions circulate in the storage ring. So, the lattice should be highly repeatable with no straight sections (e.g., no missing dipole sections). A highly symmetric lattice, as with the CRFQ, is required.

Use of longitudinal laser cooling has had success in ion traps. Transverse cooling through coupling has had some success, as well. Doppler cooling is limited by the Rabi frequency for the resonant excitation, which depends on the 
transition linewidth, excitation intensity, and the wave vector. So, another cooling system is needed, such as Raman transition cooling, to get to very low temperatures. For a quantum computer we would stay above the LambDicke limit, which will influence the amount of coupling between the internal quantum states and center-of-mass motion external quantum states [15]. Electron and laser cooling are highly efficient for longitudinal cooling, but additional systems are needed to achieve transverse cooling. A number of methods can be found in the literature, but given the range of parameters we have considered, so far, all that is clear is we need to research this more deeply. As an example, we note Eschner, Appasamy, and Toschek's research into the use of null detection in ion fluorescence has been investigated as a way to employ stochastic cooling of trapped ions [45]. This may not be practical in our case, but shows the creative methods people are developing.

Employing a system for pre-cooling before injection may prove useful, since it would enable making a finer momentum selection during injection. One method is to use a Zeeman slower/decelerator [46], where a magnetic field can change the resonance frequency using the Zeeman effect (splitting the spectral lines) to compensate for the ion momentum falling out of resonance with the optical pumping. This does have the effect of lowering the velocity of the ions.

Cooling crystalline beams as they approach a more ordered state becomes progressively more difficult. Using sympathetic cooling or coupling through a synchro-betatron resonance also is problematic as the beams approach the crystalline state [7]. Tapered cooling is one method that could provide effective 3D cooling. This method provides ions with a constant angular velocity, by causing momentum to be a function of radial displacement [47]. Tapered cooling and Wien filters are an area of active research [48].

A few other considerations, that are beyond the scope of this paper, but worth mentioning here, include, heating rates and how they depend on various parameters (e.g., is heating a function of velocity? why?), parameters that determine the laser focus (e.g., overlap of ion trajectory to laser line of sight), and methods to control or limit the transverse and longitudinal coupling.

\section{B. Timing and synchronization}

Ion crystals in an ion trap are stationary, except for the small motion due to dispersion. A unique feature of a storage ring approach is the ions are moving. As seen in Table I, given the maximum velocities for establishing an ion Coulomb crystal, each ion will traverse the circumference of the ring in roughly $1.5 \mathrm{msec}$ to over $3 \mathrm{msec}$. If we assume that a single set/read operation on a qubit can take place in $10 \mu \mathrm{sec}$, then in the storage ring an ion will still travel many millimeters in the time of a single set/read operation. This may seem to be a problem. However, since we have a ring, and can place laser beams anywhere we want, we can be exciting a large number of ions simultaneously or the same ion by a well-defined sequence. We could even design clever mirror schemes, perhaps with rotating mirrors, to keep the laser pulse on an ion as it travels past. Techniques to track the ions are already employed in ion trap systems, since they have to excite all the ions in the trap. The fact that the ions in a storage ring are moving means we do have to synchronize the laser pulses to the arrival of the ions. If we sequence the laser pulses, we can drive the qubit throughput much higher, taking advantage of parallelism in the laser arrangment.

As a simple example, if we have a $1 \mathrm{~m}$ ring filled with 100,000 ions, with $10 \mu \mathrm{m}$ spacings and $v=100 \mathrm{~m} / \mathrm{s}$, a laser pulse would have to track an ion over $1 \mathrm{~mm}$ to provide a uniform $10 \mu \mathrm{sec}$ pulse of radiation. If we cleverly distribute 1000 laser pulses around the ring, we can easily imagine exciting 1 million qubits per second. Since coherence times much greater then $1 \mathrm{sec}$ are well established, it is clear that all ions in the ring can be operated on while maintaining the $1 \mathrm{Mqubit} / \mathrm{sec}$ rate. Of course, this is with all practicalities aside. It does demonstrate, though, the potential of such an approach.

As already mentioned, we do have to be concerned with maintaining the coherence of the quantum states of the ions. A given ion may be set to a particular state but then may be ignored for a long period of time while other ions are being operated on. There will always be far more ions than lasers to set and read the states. It is always possible that something will disrupt the quantum state. This is analogous to a classical bit being upset by some external noise or radiation. While we offer no solutions here, we recognize that fault tolerance and error correction methods need to be developed to ensure the integrity of the quantum bits, just as such methods had to be developed to ensure the integrity of classical bits. This is a very active area of research [49-51] that we will pay close attention to as we develop a storage ring system. We note, though, that according to the quantum fault-tolerance theorem [52], as long as physical error rates are below a threshold, quantum error correction schemes can be used to suppress the logical error rate, enabling reliable computation. The basic idea is to correct errors faster than they occur. However, practical approaches suggest far more qubits are needed for the error correction than the actual computation (on the scale of more than 1000:1).

\section{Cooling constraints}

Two constraining parameters will determine the efficiency of the storage ring as a quantum computer, switching rate and number of ions in the ion string (which has an effective mass, $\mathrm{Nm}$ ).

As mentioned above, switching rate has to do with how the ion trap information is processed, through the use of laser pulses at a set frequency and for a set duration. In the 
case of an ion trap, the ion vibrational states have spatial extents with a probability distribution that depends on the standard deviation of those vibration amplitudes [Eq. (41)]. Vibrational state changing transitions are a function of this probability distribution as well as $1 / \sqrt{N}$ [Eq. (44)], since the string is moving as a single mass and must obey conservation of angular momentum.

The most common and simplest type of laser cooling is Doppler cooling for free ions. In this process photons excite transitions that bring the ion's internal state back to the ground state. With continued excitations, on average the vibrational quantum number is reduced. What is important for this discussion is to note that in ion traps, only a single ion is operated on by the laser. The string of ions in the trap are all being influenced, since they are coupled through the Coulomb force. In a storage ring we can use laser cooling to control independent strings of ions or to explore cooling very long strings of ions. As we mentioned in Sec. IV, since the longitudinal potentials can be operated using different frequencies we can also explore isolating chains of ions and then adiabatically merging the chains to create longer chains. Such rf harmonic gymnastics are routine in storage rings but not possible in a linear ion trap. Of course, doing this without heating the beam will be a technical issue.

A number of other cooling methods may improve on the ability to control the temperature, but a detailed survey of methods goes beyond the scope of our discussion. Methods of interest include sympethetic cooling, Sisyphus cooling, and Raman sideband cooling [53,54]. A significant challenge is transverse cooling. In an ion trap it is possible to use techniques such as optical molasses [55], to cool all degrees of freedom of the ions motional states. In a storage ring the ions are in the field of the lasers for a relatively long distance, longitudinally, but for transverse cooling the laser fields would have to track with the ion's longitudinal motion. Exposing the transverse motion to the longitudinal laser is also not very effective since the cooling will be reduced by the ratio of $v_{u}^{2} / v^{2} \approx \theta^{2}$, where $\theta$ is the angle in some chicane, for example, that exposes the transverse velocity component. However, a number of methods have been published that can be explored, such as through dispersive coupling $[31,56]$.

Raman cooling may be effective, as long as the ions are not too high in energy $(v / c \ll 1)$. Raman transitions are induced by simultaneous absorption and stimulated emission using two different lasers. For laser frequencies, $\omega_{L 1}$ and $\omega_{L 2}$, where $\omega_{L 2}$ is in the same direction as the ions trajectory, the condition for coherent resonant excitation is [55],

$$
\left(\omega_{L 1}-\omega_{L 2}\right)+\frac{v_{\text {ion }}}{c}\left(\omega_{L 1}+\omega_{L 2}\right)=\omega_{12},
$$

where $v_{\text {ion }}$ is the ion's velocity and $\hbar \omega_{12}$ is the energy difference between the two quantum levels. This process has a very narrow line width and so the laser parameters must be swept in some manner to cool the entire collection of ions. The technique only operates on ions outside of $v_{\text {ion }}$, since the parameters are selected so that ions with velocities in a narrow range around $v_{\text {ion }}$ are never excited. This technique has worked well for ions with $v_{\text {ion }} \approx 0$, but needs to be demonstrated (to our understanding) for high velocity ions. The ramifications of a nonzero velocity fall beyond the scope of our discussion, but involve what happens when the doppler shift becomes large relative to a small detuning range around the quantum levels.

\section{Entanglement}

Having an ion Coulomb crystal does not mean the ions in the crystal have entangled states. While they may share a common phonon state, the internal spin states of the ions are not in any way entangled.

Entanglement in an ion trap involves exploiting the coupling of the internal and external quantum states of the ions. Through these couplings it is possible to create quantum computer gates. The methods used to create these couplings involve exciting resonance conditions, in Raman transitions, for example, that lead to coherent interactions. In the simplest example, spin state transitions are accompanied by motional state transitions. This is done by selecting a laser frequency that is an upper or lower sideband of $\omega_{\mathrm{ph}}$, the frequency for a given external state, where spin states are separated in energy by $\hbar \omega_{\text {ph }}$. There are a number of methods for mapping from spin qubits to motion qubits that allow the motional qubit to be used as a carrier transition to create gates, such as a controlled-not (CNOT) gate [57]. Details of such schemes are well documented in the ion-trap literature [15]. In the next sections we outline some of these basic operations.

It should be noted that extremely cold ion Coulomb crystals are really only needed if we are trying to exploit the lower frequency axial phonon modes. Recent work exploiting higher frequency transverse modes [30] promises to allow scaling to a higher number of entangled ions. Other techniques, such as using photons that are entangled with the ion qubits may not require the beams to be cooled so close to the Lamb-Dicke regime [58].

\section{METHODS TO EXTRACT QUANTUM INFORMATION}

The primary method of establishing a qubit involves excitation and measurement of hyperfine states [58]. As described by Wineland et al., using ${ }^{9} \mathrm{Be}^{+}$and using the ${ }^{2} S_{1 / 2}\left(F=2, m_{F}=2\right)$ and ${ }^{2} S_{1 / 2}\left(F=1, m_{F}=1\right)$ hyperfine ground states (denoted $|\downarrow\rangle$ and $|\uparrow\rangle$, respectively), a practical qubit can be constructed [15]. A polarized laser beam is tuned to the $|\downarrow\rangle \rightarrow{ }^{2} P_{3 / 2}$ transition and by observing the scattered photons two distinct spin states can be resolved. With this technique, per Wineland et al., the quantum states can be determined with almost 100\% efficiency [58]. 
Another form of quantum information can be observed in the axial center-of-mass motion of the ions, as discussed above. This axial motion has a frequency $\omega_{z}$, which is independent of the number of ions in the trap and is described by the vibrational eigenstates $|n\rangle$ with energy $\hbar \omega_{z}\left(n+\frac{1}{2}\right)$, where $n$ is the vibrational index describing the number of phonons in the collective harmonic motion. The axial frequency, $\omega_{z}$, is a function of the trap potential, the dimensions of the trap, and the charge and mass of the trapped ion. To create quantum operations, internal energy eigenstates and center-of-mass motion are employed. Internal energy eigenstates are measured by observing the frequency of the radiation resulting from the technique of quantum jumps [59]. For example, the two internal energy eigenstates $|0,0\rangle$ and $|1,0\rangle$ are separated in frequency by $\hbar \omega_{v}$. Each of these internal states has an associated set of vibrational levels for each vibrational mode. The vibrational energy eigenstates are $\mid n_{1}, n_{2}$, $\left.n_{3}, \ldots\right\rangle$, where $n_{i}$ are the excitations of the various normal modes. The ground state is $|0,0,0, \ldots\rangle$ and the first excited state of the center-of-mass is $|1,0,0, \ldots\rangle$. These then become the basis of the computation operations. Observing axial modes in an ion trap is done through coupling between internal and external vibration states by stimulated Raman transitions.

\section{TOWARD A QUANTUM COMPUTER}

In Secs. V to VII, we have discussed an example of a storage ring that was able to create crystalline beams and the parameters needed to develop ion Coulomb crystals useful for quantum computing. While many of the ideas presented are ambitious, our goal has been to explore what may be possible given we use a storage ring as an ion trap rather than a traditional linear ion trap.

To actually develop a working quantum computer using an ion Coulomb crystal in a storage ring, there must be at least two components to the system; a method to set the initial states and an ability to operate on the quantum information [22,60-62].

\section{A. Initialization}

An ion trap system is able to set the internal state of the ions to $|\downarrow\rangle$ by utilizing optical pumping techniques as described above. Applying this to all ions in the storage ring may be challenging, due to the large number of ions in the beam. But, as already discussed, this is something that can be managed in how the crystals are formed and controlled.

\section{B. Quantum gates}

A gate that acts on $i$ qubits is represented by a $2^{i} \times 2^{i}$ unitary matrix [63]. A vector representation of a qubit from an internal spin state is,

$$
u_{0}|\downarrow\rangle+u_{1}|\uparrow\rangle \rightarrow\left[\begin{array}{l}
u_{0} \\
u_{1}
\end{array}\right]
$$

A single bit rotation gate, operator $R(\theta, \phi)$ transforms the state of the qubit as,

$$
\begin{aligned}
& |\downarrow\rangle \rightarrow \cos (\theta)|\downarrow\rangle-i e^{i \phi} \sin (\theta)|\uparrow\rangle \\
& |\uparrow\rangle \rightarrow \cos (\theta)|\uparrow\rangle-i e^{-i \phi} \sin (\theta)|\downarrow\rangle
\end{aligned}
$$

This transformation can be realized in a storage ring, for a single bit rotation gate, by tuning the frequency $\left(\omega_{L}=\omega_{0}\right)$ and radiating for a time $t$ such that $\theta=2 \Omega^{j} t$, for the $j$ th ion in the string, where $\Omega^{j}$ is the Rabi frequency of $|\downarrow\rangle_{j}$ to $|\uparrow\rangle_{j}$. In this way an ion first prepared to state $|\downarrow\rangle$ can be measured in the state $|\downarrow\rangle$ or $|\uparrow\rangle$.

Realizing quantum gates for a storage ring quantum computer is largely an independent topic and beyond the scope of this report. In this section we wished to only present how the techniques already developed for ion trap systems would still apply in a storage ring system.

\section{Quantum memory}

Ion Coulomb crystals are not just useful as computational objects, but also as memory objects [64]. Since the states of the qubits can be controlled, states from one crystal can be written to another crystal. The final state (either $|\uparrow\rangle$ or $|\downarrow\rangle$ ) is known from the fluorescence of the ions. The rotation angle $(\theta)$ and phase information $(\phi)$ can be determined from the carrier drive duration and the difference between the upper band and lower band of the Raman nonresonant laser pulses. The details of this technique are well described by Kielpinski et al. [64]. However, the no-cloning theorem $[65,66]$ states that we cannot keep the original quantum state, we can only transfer it to another ion. Nevertheless, the ability to perform this operation is clearly very useful.

Having multiple crystals in the storage ring provides the potential to choose how each crystal is being utilized. By having many ion crystals we can start to imagine the one system being usable as a single large-scale quantum computational system.

\section{CONCLUSIONS}

We have introduced the idea of using storage rings with crystalline beams as quantum computers. There are a number of phenomena that could be exploited to produce quantum bits of information. The challenge in building such a computer hinges entirely on how effectively particle beams can be cooled to a sufficient level and on how quickly and reliably the quantum states can actually be set and measured. The benefit of this approach is it allows exploring large numbers of ions and qubits under well controlled and isolated conditions. Conceptually, such a 
system is the same as an ion trap quantum computer, but with the ions placed in the rotating frame in the circular storage ring.

\section{ACKNOWLEDGMENTS}

This work was performed under Contract No. DESC0012704 with the auspices of the U.S. Department of Energy.

[1] S. van der Meer, Frontiers of Particle Beams; Observation, Diagnosis and Correction, Diagnostics with schottky noise (Springer Berlin/Heidelberg, 1989), pp. 423-433.

[2] S. Chattopadhyay, Some fundamental aspects of fluctuations and coherence in charged-particle beams in storage rings, AIP Conf. Proc. 127, 467 (1985); Reports No. CERN-84-11, No. CERN-YELLOW-84-11.

[3] D. Boussard, Schottky noise and beam transfer function diagnostics, Proceedings v 2, CAS CERN Accelerator school-Advanced accelerator physics, Oxford, edited by S. Turner (CERN, Geneva, 1985), pp. 416-452 [Report No. CERN 87-03 v 2.

[4] K. A. Brown, M. Blaskiewicz, C. Degen, and A. Della Penna, Measuring transverse beam emittance using a $2.07 \mathrm{GHz}$ movable Schottky cavity at the Relativistic Heavy Ion Collider, Phys. Rev. Accel. Beams 12, 012801 (2009).

[5] J. Struckmeier, Concept of entropy in the realm of charged particle beams, Phys. Rev. E 54, 830 (1996).

[6] J. D. Lawson, P. M. Lapostolle, and R. L. Gluckstern, Emittance, entropy and information, Part. Accel. 5, 61 (1973).

[7] J. Wei and A. M. Sessler, Crystalline beams, 18th Advanced ICFA Beam Dynamics Workshop on Quantum Aspects of Beam Physics, edited by P. Chen, 2003, p. 182 [Brookhaven National Laboratory Report No. SLACReport-574].

[8] A. M. Sessler, Methods of beam cooling, 31st Workshop on Crystalline Beams and Related Issues, Erice, Italy, November, 1995 [Lawrence Berkeley Laboratory Report No. LBL-38278].

[9] T. Schätz, U. Schramm, and D. Habs, Crystalline ion beams, Nature (London) 412, 717 (2001).

[10] A. Steane, The ion trap quantum information processor, Appl. Phys. B 64, 623 (1997).

[11] D. J. Wineland, Experimental issues in coherent quantumstate manipulation of trapped ions, J. Res. Nat. Inst. Standards Technol. 103, 259 (1998).

[12] J. Schmidt, A. Lambrecht, P. Weckesser, M. Debatin, L. Karpa, and T. Schätz, Optical Trapping of Ion Coulomb Crystals, Phys. Rev. X 8, 021028 (2018).

[13] M. Asprusten, S. Worthington, and R.C. Thompson, Theory and simulation of ion Coulomb crystal formation in a Penning trap, Appl. Phys. B 114, 157 (2014).

[14] A. Mortensen, E. Nielsen, T. Matthey, and M. Drewsen, Observation of Three-dimensional Long-range Order in Smaller Ion Coulomb Crystals in an rf Trap, Phys. Rev. Lett. 96, 103001 (2006).
[15] D. J. Wineland, C. Monroe, W. M. Itano, B. E. King, D. Leibfried, D. M. Meekhof, C. Myatt, and C. Wood, Experimental primer on the trapped ion quantum computer, Fortschr. Phys. 46, 363 (1998).

[16] M. Schlosshauer, Decoherence, the measurement problem, and interpretations of quantum mechanics, Rev. Mod. Phys. 76, 1267 (2005).

[17] D. Bacon, Decoherence, control, and symmetry in quantum computers, arXiv:quant-ph/0305025.

[18] B. Tabakov, F. Benito, M. Blain, C. R. Clark, S. Clark, R. A. Haltli, P. Maunz, J. D. Sterk, C. Tigges, and D. Stick, Assembling a ring-shaped crystal in a microfabricated surface ion trap, Phys. Rev. Applied 4, 031001 (2015).

[19] D. Kielpinski, C. Monroe, and D. J. Wineland, Architecture for a large-scale ion-trap quantum computer, Nature (London) 417, 709 (2002).

[20] W. Paul, Electromagnetic traps for charged and neutral particles, Rev. Mod. Phys. 62, 531 (1990).

[21] U. Schramm and D. Habs, Crystalline ion beams, Prog. Part. Nucl. Phys. 53, 583 (2004).

[22] D. P. DiVincenzo, The physical implementation of quantum computation, Fortsch. Phys. 48, 771 (2000).

[23] W. Paul, Electromagnetic traps for charged and neutral particles, Rev. Mod. Phys. 62, 531 (1990).

[24] D. G. Cory, A. F. Fahmy, and T. F. Havel, Ensemble quantum computing by NMR spectroscopy, Proc. Natl. Acad. Sci. U.S.A. 94, 1634 (1997).

[25] N. A.Gershenfeld and I. L. Chuang, Bulk spin-resonance quantum computation, Science 275, 350 (1997).

[26] L. M. Vandersypen, M. Steffen, G. Breyta, C. S. Yannon, M. H. Sherwood, and I. L. Chuang, Experimental realization of Shor's quantum factoring algorithm using nuclear magnetic resonance, Nature (London) 414, 883 (2001).

[27] N. C. Menicucci and C. M. Caves, Local Realistic Model for the Dynamics of Bulk-Ensemble NMR Information Processing, Phys. Rev. Lett. 88, 167901 (2002).

[28] J. A. Jones, NMR quantum computation: A critical evaluation, Fortschr. Phys. 48, 909 (2000).

[29] L. Gyongyosi and S. Imre, A Survey on quantum computing technology, Comput. Sci. Rev. 31, 51 (2019).

[30] S. Zhu, C. Monroe, and L.-M. Duan, Trapped Ion Quantum Computation with Transverse Phonon Modes, Phys. Rev. Lett. 97, 050505 (2006).

[31] I. Lauer, U. Eisenbarth, M. Grieser, R. Grimm, P. Lenisa, V. Luger, T. Schätz, U. Schramm, D. Schwalm, and M. Weidemüller, Transverse Laser Cooling of a Fast Stored Ion Beam through Dispersive Coupling, Phys. Rev. Lett. 81, 2052 (1998).

[32] R. W. Hasse, Theoretical Verification of Coulomb Order of Ions in a Storage Ring, Phys. Rev. Lett. 83, 3430 (1999).

[33] R. W. Hasse, Static Criteria for the Existence of Coulomb Strings in Storage Rings, Phys. Rev. Lett. 90, 204801 (2003).

[34] J.P. Schiffer and P. Kienle, Could there be an ordered condensed state in beams of fully stripped heavy ions, $\mathrm{Z}$. Phys A - Atoms and Nuclei 321, 181 (1985).

[35] A. Rahman and J. P. Schiffer, Structure of a One-Component Plasma in an External Field: A Molecular-Dynamics Study 
of Particle Arrangement in a Heavy-Ion Storage Ring, Phys. Rev. Lett. 57, 1133 (1986).

[36] M. Tanabe, T. Ishikawa, M. Nakao, H. Souda, M. Ikegami, T. Shirai, H. Tongu, and A. Noda, Longitudinal and transverse coupling of the beam temperature caused by the laser cooling of related content, Appl. Phys. Express 1, 028001 (2008).

[37] U. Schramm, T. Schätz, M. Bussmann, and D. Habs, The quest for crystalline ion beams, Plasma Phys. Controlled Fusion 44, B375 (2002).

[38] U. Schramm, T. Schätz, M. Bussmann, and D. Habs, Cooling and heating of crystalline ion beams, J. Phys. B 36, 561 (2003).

[39] U. Schramm, T. Schätz, M. Bussmann, and D. Habs, Storage of crystalline ion beams, in Proceedings of the 2003 Particle Accelerator Conference, Portland, OR (IEEE, New York, 2003), TOAA004, http://www.jacow .org.

[40] U. Schramm, T. Schätz, and D. Habs, Bunched Crystalline Ion Beams, Phys. Rev. Lett. 87, 184801 (2001).

[41] U. Schramm, T. Schätz, and D. Habs, Three-dimensional crystalline ion beams, Phys. Rev. E 66, 036501 (2002).

[42] A. Noda et al., Laser cooling for 3-D crystalline state at L-LSR, AIP Conf. Proc. 821, 491 (2006).

[43] A. G. Ruggiero, The circular RFQ storage ring, BNL Internal Report No. BNL-104887-2014-TECH, 1998 (to be published).

[44] M. Steck, K. Beckert, H. Eickhoff, B. Franzke, F. Nolden, H. Reich, B. Schlitt, and T. Winkler, Phys. Rev. Lett. 77, 3803 (1996).

[45] J. Eschner, B. Appasamy, and P. E. Toschek, Stochastic Cooling of a Trapped Ion by Null Detection of Its Fluorescence, Phys. Rev. Lett. 74, 2435 (1995).

[46] B. Ohayon and G. Ron, New approaches in designing a Zeeman Slower, J. Instrum. 8, P02016 (2013).

[47] H. Okamoto and J. Wei, Theory of tapered cooling, Phys. Rev. E 58, 3817 (1998).

[48] A. Noda et al., Experimental strategy for realization of 3-D beam ordering with use of tapered cooling at S-SLR, Proceedings of HB2006, WEAY06 (2006) http://www .jacow.org.

[49] J. M. Auger, H. Anwar, M. Gimeno-Segovia, T. M. Stace, and D. E. Browne, Fault-tolerant quantum computation with nondeterministic entangling gates, Phys. Rev. A 97, 030301(R) (2018).

[50] Y.-C. Zheng, C.-Y. Lai, and T. A. Brun, Efficient preparation of large-block-code ancilla states for fault-tolerant quantum computation, Phys. Rev. A 97, 032331 (2018).

[51] K. Fukui, A. Tomita, A. Okamoto, and K. Fujii, HighThreshold Fault-Tolerant Quantum Computation with
Analog Quantum Error Correction, Phys. Rev. X 8, 021054 (2018).

[52] D. Aharonov and M. Ben-Or, Fault-tolerant quantum computation with constant error rate, arXiv:quant-ph/ 9906129; in Proceedings of the twenty-ninth annual ACM symposium on Theory of computing (STOC '97) (ACM, New York, 1997), pp. 176-188.

[53] H. J. Metcalf and P. van der Straten, Laser Cooling and Trapping (Springer, New York, 1999), Sect. 8.8, ISBN 9780387987286.

[54] M. Kasevich and S. Chu, Laser Cooling below Photon Recoil with Three-Level Atoms, Phys. Rev. Lett. 69, 1741 (1992).

[55] C. J. Foot, Atomic Physics (Oxford University Press, New York, 2014), ISBN 978-0-19-850696-6.

[56] H. Okamoto, Transverse laser cooling induced through dispersion at an rf cavity, Phys. Rev. E 50, 4982 (1994).

[57] J. I. Cirac and P. Zoller, Quantum Computations with Cold Trapped Ions, Phys. Rev. Lett. 74, 4091 (1995).

[58] D. Wineland, Nobel Lecture: Superposition, entanglement, and raising Schrödinger's cat, Rev. Mod. Phys. 85, 1103 (2013).

[59] W. Nagourney, J. Sandberg, and H. G. Dehmelt, Shelved Optical Electron Amplifier: Observation of Quantum Jumps, Phys. Rev. Lett. 56, 2797 (1986); T. Sauter, W. Neuhauser, R. Blatt, and P.E. Toschek, Observation of Quantum Jumps, Phys. Rev. Lett. 57, 1696 (1986); J. C. Bergquist, R. G. Hulet, W. M. Itano, and D. J. Wineland, Observation of Quantum Jumps in a Single Atom, Phys. Rev. Lett. 57, 1699 (1986).

[60] P. Benioff, The computer as a physical system: A microscopic quantum mechanical Hamiltonian model of computers as represented by Turing machines, J. Stat. Phys. 22, 563 (1980).

[61] Yu. I. Manin, Vychislimoe i nevychislimoe, Computable and Noncomputable (in Russian), Sov. Radio., 13 (1980). Archived from the original on 2013-05-10.

[62] R. P. Feynman, Simulating physics with computers, Int. J. Theor. Phys. 21, 467 (1982).

[63] M. Nielsen and I. Chuang, Quantum Computation and Quantum Information (Cambridge University Press, Cambridge, England, 2000), ISBN 0-521-63503-9. OCLC 174527496.

[64] D. Kielpinski, V. Meyer, M. A. Rowe, C. A. Sackett, W. M. Itano, C. Monroe, and D. J. Wineland, A decoherence-free quantum memory using trapped ions, Science 291, 1013 (2001).

[65] W. K. Wootters and W. H. Zurek, A single quantum cannot be cloned, Nature (London) 299, 802 (1982).

[66] D. Dieks, Communication by EPR devices, Phys. Lett. A 92, 271 (1982). 